\title{
Covering an Arbitrary Shaped Domain by Identical Circles
}

\author{
Alexandr Pankratov \\ Department of Mathematical Modeling \\ and Optimal Design \\ Institute for Mechanical Engineering \\ Problems of the National Academy of \\ Sciences of Ukraine \\ Kharkiv, Ukraine \\ pankratov2001@yahoo.com \\ Yuliia Pankratova \\ Department of Systems Engineering \\ Kharkiv National University of Radio \\ Electronics \\ Kharkiv, Ukraine \\ yuliia.pankratova@nure.ua
}

\author{
Tatyana Romanova \\ Department of Mathematical Modeling \\ and Optimal Design \\ Institute for Mechanical Engineering \\ Problems of the National Academy of \\ Sciences of Ukraine \\ Kharkiv, Ukraine \\ tarom27@yahoo.com \\ Sergiy Shekhovtsov \\ Department of Information \\ Technologies \\ National University of Internal Affairs \\ Kharkiv, Ukraine \\ ep109@ukr.net
}

\author{
Oleksiy Antoshkin \\ Department of Automated Security \\ Systems and Information Technologies \\ National University of Civil Protection \\ of Ukraine \\ Kharkiv, Ukraine \\ antoshkin@nuczu.edu.ua
}

\author{
Vadim Kartak \\ Department of Computer Science and \\ Robotics
}

Ufa State Aviation Technical University

Ufa, Russia

kvmail@mail.ru

\begin{abstract}
The problem of covering a bounded disconnected arbitrary shaped area (domain) by identical circles is considered. To describe analytically the coverage conditions we use special continuous and everywhere defined functions for modelling relations between circles and the border of the area. A new function for modelling the relations between three circles when covering the interior part of the domain is defined. An integrated mathematical model of the coverage problem is provided in the form of a nonlinear programming problem. A new strategy for solving the problem is proposed. To demonstrate the efficiency of the developed algorithm an example of solving the problem for optimizing the length of the network connecting centers of the circles is presented.
\end{abstract}

Keywords-circular coverage, arbitrary shaped domain, identical circles, tracing, mathematical model, nonlinear optimization.

\section{INTRODUCTION}

Currently, there is a rapidly growing interest in the effective solution of the problems of optimum coverage of areas with circles known as the problems of circular coverage (see, e.g., $[1,2])$. One of the interesting application of coverage problems in the geometric design systems is considered in $[3,4]$

This study is motivated by a variety of practical applications including the construction of network, the protection of forest arrays from fires, determining the necessary quantity and arrangement of the stations of cellular connection, determining the operation range and arrangement of watering plants, intended for controlling the range of circular orbits of artificial Earth satellites. The optimum coverage of domains relates to NP-complex problems. Therefore, in most publications heuristic algorithms are used for their solution. Thus, developing efficient algorithms for the coverage problems requires mathematical models based on the analytical description of relations between the objects.

We present here new tools for the analytical description of the coverage relations of the inner part of the domain and propose a new four-stage solution strategy for the problem. Algorithms for generating the objective function to optimize the length of the network for two types of sensor networks are provided. In addition some computational results are introduced.

\section{RELATED PAPERS AND PROBLEM FORMULATION}

Many studies are devoted to the coverage problems [1, 2]. As follows from [2], some heuristics for circular coverage is the arrangement of sensors in determined units according to some template. The more advanced approaches employ the Voronoi diagrams [5] and the Delaunay [6] triangulation.

Some heuristics are based on the random arrangement of sensors in the covered domain [7]. In this case two subproblems appear: 1) is the given domain fully covered by the circles? and 2) eliminate the excessive sensors. The basic techniques of solving these sub-problems are highlighted in [2]. if

The most efficient coverage criterion is proposed in [8]:

1) each interior point of the domain that is a point of the intersection of the frontiers of two circles belongs to at least one of the rest part of the circles;

2) each point of the intersection of circles and the frontier of the domain belongs to at least one of the rest part of the circles.

Metaheuristics are frequently used to improve the random arrangement of circles, e.g., ants' colony algorithms [9], genetic algorithms [10], simulation annealing [11].

Only a few works are devoted to the construction of exact mathematical models for the coverage problems.

Authors of [12] propose the approach to cover a square by single circles, based on the theory of temperature expansions and compressions of pivotal structures. The approach, proposed in [13], uses the Dirichlet-Voronoi scheme. The results are presented for a single square. 
Article [14] considers the continuous problem of set coverage as the problem of quasi-differentiable optimization. Authors of [15] propose the algorithm based on the theory of the optimum division of sets and the Shor's r-algorithm [16]. However, the proposed in [15] algorithm dependences on the parameters of the algorithm: the magnitude of step of the three-dimensional grid and magnitude of step of numerical differentiation with the calculation of the components of the generalized gradient.

In [17] the numerical algorithm of solving circular coverage of arbitrary convex domains is based on the use of sub-differential and sub-gradient methods.

In [18] authors study the problem of coverage of polygons by identical circles, construct the mathematical model on the basis of the Voronoi diagram. The mathematical model of the problem of coverage of an arbitrary polygon with non-identical circles is developed in [19] using the coverage criterion, proposed in [8].

Based on the elaboration of the approach described in [19], a mathematical model of the problem of covering an arbitrary domain with identical circles is proposed in [20]. The model uses phi-functions [21] and quasi phi-functions [22].

The main contributions of our paper are follows:

- further development of geometric tools for analytical description of relations between objects in the circular coverage problems;

- development of the integrated mathematical model of circular coverage with identical circles in the form of the non-smooth optimization problem combining with the optimization of the trace routing connections;

- development of algorithms to search for local-optimal solutions.

\section{MATHEMATICAL MODEL AND SOLUTION ALGORITHM}

\section{Mathematical model}

Let $\Omega \subset R^{2}$ be a closed bounded area with the piecewise-smooth boundary, formed by $\mathrm{L}$ fragments of curves. Then we assume that a collection of identical circles $C=\left\{C_{i}, i=1, \ldots, n\right\}$ of radius $r$ with the center points $u_{i}$ is given. The set $\Upsilon=\bigcup_{i=1}^{n} C_{i}$ is called the circular coverage of area $\Omega$, if $\Omega \subseteq \Upsilon$.

Problem formulation. Find a coverage $\Upsilon$ of the domain $\Omega$, to optimize an objective function $F(u)$, $u=\left(u_{1}, u_{2}, \ldots, u_{n}\right)$.

It should be noted that $F(u)$ is a smooth function.

First we form the set $P$ of the points $p_{k}, k=1, \ldots, K$ in which the smoothness of the frontier of $\Omega$ is disrupted. In what follows, we assume that the curvature of the boundary at any point, except points from the set $\mathrm{P}$, is less than the curvature of circles from the set $\mathrm{C}$.
For the set $\Upsilon(u)=\bigcup_{i=1}^{n} C_{i}\left(u_{i}\right)$ to be the non-degenerate [20] circular coverage of $\Omega$, it is necessary and sufficient that:

1) for each point $p_{k} \in P$ at least one circle $C_{i}\left(u_{i}\right)$, $i \in I_{n}$ should be found so that $p_{k} \in \operatorname{int} C_{i}\left(u_{i}\right)$;

2) for any point $t_{i k} \in f r C_{i}\left(u_{i}\right) \bigcap f r \Omega, i \in I_{n}$ at least one circle $C_{j}\left(u_{j}\right), j \in I_{n}, i \neq j$, should be found so that $t_{i k} \in \operatorname{int} C_{j}\left(u_{j}\right) \quad$ and, consequently, point $t_{i j k} \in \operatorname{fr} C_{i}\left(u_{i}\right) \cap \operatorname{fr} C_{j}\left(u_{j}\right)$ should belong to $\Omega^{*}=R^{2} \backslash \operatorname{int} \Omega$

3) for any point $t_{i j k} \in \operatorname{fr} C_{i}\left(u_{i}\right) \cap \operatorname{fr} C_{j}\left(u_{j}\right), i \neq j$, $i, j \in I_{n}, t_{i j k} \in \operatorname{int} \Omega, k \in\{1,2\}$, at least one circle $C_{s}\left(u_{s}\right)$, $s \in I_{n}, s \neq i, s \neq j$ should be found so that $t_{i j k} \in \operatorname{int} C_{s}\left(u_{s}\right)$

The coordinates of points, $t_{i j k}, k \in\{1,2\}$ of intersection of $\operatorname{frC}_{i}\left(u_{i}\right)$ and $\operatorname{frC}_{j}\left(u_{j}\right)$ are derived by the formulas

$$
\begin{gathered}
x_{i j 1}=\frac{x_{i}+x_{j}}{2}+d\left(y_{j}-y_{i}\right) \quad, \quad y_{i j 1}=\frac{y_{i}+y_{j}}{2}-d\left(x_{j}-x_{i}\right), \\
x_{i j 2}=\frac{x_{i}+x_{j}}{2}-d\left(y_{j}-y_{i}\right) y_{i j 2}=\frac{y_{i}+y_{j}}{2}+d\left(x_{j}-x_{i}\right),
\end{gathered}
$$

where

$$
d=\sqrt{\frac{r^{2}}{\left(x_{j}-x_{i}\right)^{2}+\left(y_{j}-y_{i}\right)^{2}}-0.25}
$$

The second and the third criteria are reformulated and auxiliary variables are introduced in order to avoid redundant calculations [19]. We use the simple way to derive coordinates of points $t_{i j k}$ analytically.

Mathematical model of the circular coverage problem can be presented in the form

$$
\underset{u \in W \subset R^{\delta}}{\operatorname{extr}} F(u, t)
$$

$$
\begin{aligned}
& W=\left\{(u, t) \in R^{\delta}: \phi^{p_{k} C_{i}}\left(u_{i}\right) \geq 0,(i, k) \in \Xi_{1},\right. \\
& \min \left\{\phi^{t_{i j k} \Omega^{*}}\left(u_{i}, u_{j}\right), \Phi^{C_{i} C_{j}}\left(u_{i}, u_{j}\right),(i, j, k) \in \Xi_{2}\right\} \geq 0, \\
& \min \left\{F_{i j s}^{k}\left(u_{i}, u_{j}, u_{s}\right), \Phi^{C_{i} C_{j}}\left(u_{i}, u_{j}\right),\right. \\
& \left.\left.(i, j, s, k) \in \Xi_{3}\right\} \geq 0, \Psi(u, t) \geq 0\right\},
\end{aligned}
$$

where $(u, t)$ is a vector of the problem variables;

$F(u, t)$ is a smooth function;

$\Xi_{1}, \Xi_{2}$ and $\Xi_{3}$ are index sets that provide the appropriate coverage conditions 1)-3) [20].

$\sigma=2 n+l$ is the problem dimension;

$l$ is the number of auxiliary variables; 
$t$ is a vector of auxiliary variables;

$\varphi^{p_{k} C_{i}}\left(u_{i}\right)$ is responsible for belonging the point $p_{k}$ to the circle $C_{i}$

$\varphi^{t_{i j k} \Omega^{*}}\left(u_{i}, u_{j}\right)$ is responsible for belonging the point $t_{i j k}$ to the object $\Omega^{*}$;

$F_{i j s}^{k}\left(u_{i}, u_{j}, u_{s}\right)$ is responsible for the covering of the inner part of the domain by three circles $C_{i}, C_{j}$ and $C_{s}$;

$$
\Phi^{C_{i} C_{j}}\left(u_{i}, u_{j}\right)=4 r^{2}-\left(x_{i}-x_{j}\right)^{2}-\left(y_{i}-y_{j}\right)^{2} \quad \text { is the }
$$
adjusted phi-function of a pair of circles $C_{i}$ and $C_{j}$ providing the maximum allowable distance $\rho=0$;

$\Psi(u, t) \geq 0$ is the inequality system for describing the placement constraints of sensors as physical objects.

Function $F_{i j s}^{k}$ has the form

$$
\begin{aligned}
& F_{i j s}^{1}\left(u_{i}, u_{j}, u_{s}\right)=r^{2}-\left(\frac{x_{i}+x_{j}}{2}-x_{s}+d\left(y_{i}-y_{j}\right)\right)^{2}- \\
& \left(\frac{y_{i}+y_{j}}{2}-y_{s}-d\left(x_{j}-x_{i}\right)\right)^{2}, \\
& F_{i j s}^{2}\left(u_{i}, u_{j}, u_{s}\right)=r^{2}-\left(\frac{x_{i}+x_{j}}{2}-x_{s}-d\left(y_{i}-y_{j}\right)\right)^{2}- \\
& \left(\frac{y_{i}+y_{j}}{2}-y_{s}+d\left(x_{j}-x_{i}\right)\right)^{2},
\end{aligned}
$$

where $d$ is defined by (1).

Below we list important practical applications for the problem (2) - (3)

- minimizing lengths of wired network;

- minimizing the radius of the covering circles;

- minimizing the number of circles in a circular coverage;

- optimizing the coverage density;

- correcting placement of circles for invalid coverage;

- correcting the invalid placement of sensors (as physical objects).

In the general case problem (2)-(3) is the non-smooth optimization problem since $\varphi^{t_{i j k} \Omega^{*}}\left(u_{i}, u_{j}\right)$ can be a minimax function. The solution of the problem can be reduced to solving a sequence of nonlinear programming problems using the approach described in [21].

\section{Solution strategy}

Taking into account the features of (2)-(3), we propose the solution strategy based on the multistart method [23] with following steps.
- Step 1. Generate the set of feasible starting points of problem (2)-(3).

- Step 2. Construct system of nonlinear constraints and objective function of problem (2)-(3) using the points, obtained at Step 1.

- Step 3. Search for the local extremum of problem (2)(3), starting from the feasible points, obtained at Step 1.

- Step 4. Choose the best of the obtained at Step 3 local extrema as an approximation to the global solution of the problem (2)-(3).

\section{Solution algorithms}

For generating feasible starting circular coverage at the first step of our strategy we employ the grid-based regular coverage algorithm, the stochastic algorithm based on the homothetic transformation of circles [18], and the optimization by groups of variables.

To formulate the problem of optimizing the network length we add to system of inequalities $\Psi(u, t) \geq 0$ the following constraints:

- for belonging the sensors to the domain $\Omega$, taking into account the minimum allowable distances to the frontier of $\Omega$ using non-smooth phi-functions;

- for non-belonging of the sensor centers to the prohibition areas using non-smooth phi-functions;

- for describing minimum allowable distances between sensor centers, using smooth phi-functions

- Then we construct a network before proceeding with the formalization of the objective function.

Two basic forms of wire connections are used in practice: the annular connection with a large quantity of sensors and the plume connection, when several plumes with the limited quantity of sensors on each of them can begin at one starting point. It is desirable to obtain the minimum length of the wire connections. The first problem is the salesman problem. The second problem can be considered as the vehicle routing problem (without returning to the starting point).

We use the VPRH library [24] to solve these problems. VRPH is written in $\mathrm{C}++$ with the open initial code. It employs heuristics for solving the problem of vehicle routing (and as a special case, the salesman problem). The library has been thoroughly checked by many researchers on benchmark problems and, on the average, it returns the solution in the limits of $3 \%$ from the optimum. The library can be easily modified to include auxiliary restrictions. We modified the code to select the preferred direction (vertical, horizontal or lack of preference) for the routes, which allows us to increase the solution efficiency.

Let the networks connecting the centers of circles be obtained as a result of solving the corresponding salesman and vehicle routing problems.

For the annular type of wired connections, the objective function can be defined as 
$\rho\left(u_{0}, u_{m_{1}}\right)+\sum_{i=1}^{n-1} \rho\left(u_{m_{i}}, u_{m_{i+1}}\right)+\rho\left(u_{m_{n}}, u_{0}\right)$

$m_{i} \in\{1,2, \ldots, n\}$ is the circle numbers, $m_{i} \neq m_{j}, \quad i \neq j$; $\sum_{i=1}^{n-1} \rho\left(u_{m_{i}}, u_{m_{i+1}}\right)$ is the sum of the distances between the centers of the circles taken in an order $m_{1}, m_{1}, \ldots, m_{n}$; $\rho\left(u_{0}, u_{m_{1}}\right)$ and $\rho\left(u_{m_{n}}, u_{0}\right)$ are the distances from the starting point $u_{0}$ of the route to the centers of $C_{m_{i}}$ and $C_{m_{j}}$ respectively.

For the plume type of wired connections, the objective function takes the form $\sum_{q=1}^{Q}\left(\rho\left(u_{0}, u_{m_{1}^{q}}\right)+\sum_{i=1}^{n_{q}-1} \rho\left(u_{m_{i}^{q}}, u_{m_{i+1}^{q}}\right)\right)$, where $Q$ is the number of plumes; $m_{i}^{q} \in\{1,2, \ldots, n\}$ is the number of circles, $m_{i}^{q} \neq m_{j}^{q}, i \neq j, q=1,2, \ldots, Q, m_{i}^{q_{1}} \neq m_{j}^{q_{2}}$ $q_{1} \neq q_{2}, q_{1}, q_{2}=1,2, \ldots, Q,^{\sum_{q=1}^{Q} n_{q}=n} ; \sum_{i=1}^{n_{q}-1} \rho\left(u_{m_{i}^{q}}, u_{m_{i+1}^{q}}\right)$ is the sum of the distances between the centers of the circles taken in the order $m_{1}^{q}, m_{2}^{q}, \ldots, m_{n_{q}}^{q} ; \rho\left(u_{0}, u_{m_{1}^{q}}\right)$ is the distance from the starting point $u_{0}$ of the route to the center of the first circle in $q$, s plume.

We reduce the problem (2)-(3) to the sequence of subproblems of nonlinear programming. The search for the local extrema is carried out with the help of IPOPT [25].

\section{COMPUTATIONAL Results}

To demonstrate the efficiency of the proposed strategy, we represent an example of solving the problem of minimizing the length of the network connecting the centers of the covering circles.

Figures 1-4 show the optimized network for 57 circles 1) constructed using the VRPH for connection of annular type, length is 630.87, running time is 0.81 sec. (Fig.1); 2) constructed using the VRPH for connection of plume type, length is 6124.28, running time is 1.01 sec. (Fig.2); 3) obtained as a result of optimizing connection of annular type, length is 590.38, running time is 4.18 sec. (Fig. 3); 4) obtained as a result of optimizing connection of plume type, length is 571.58, running time is $3.42 \mathrm{sec}$. (Fig. 4). Computational experiments were carried out using AMD Athlon(tm) 64x2 Dual Core Processor 5200+.

\section{CONCLUSION}

A mathematical model of the problem of covering an arbitrary shaped domain with identical circles based on the new geometrical tools is introduced. The four-stage solution strategy for the coverage problem is developed. The challenging computational results for the problem of minimizing the length of the network connecting the centers of the covering circles are provided.

\section{ACKNOWLEDGMENT}

The reported study was funded by RFBR, project number 19-07-00895-a.

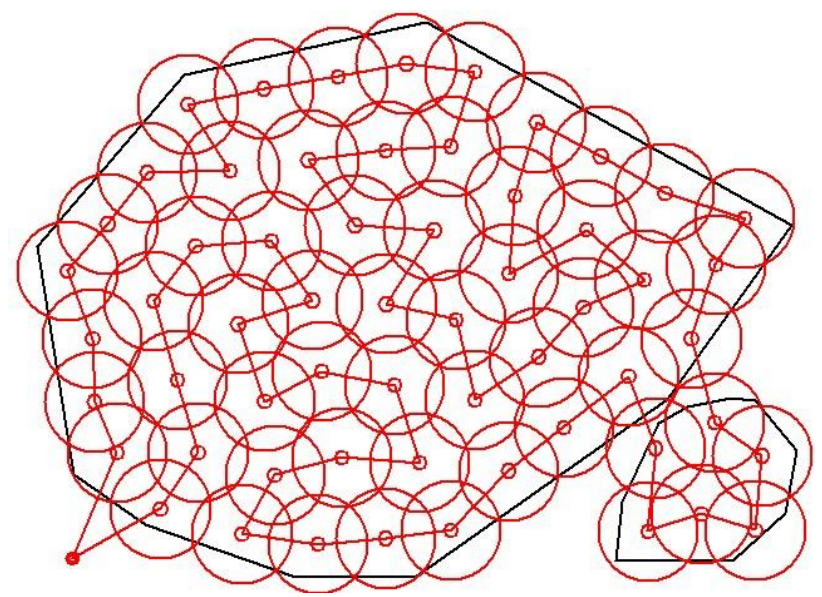

Fig. 1. Networks for 57 circles (case 1)

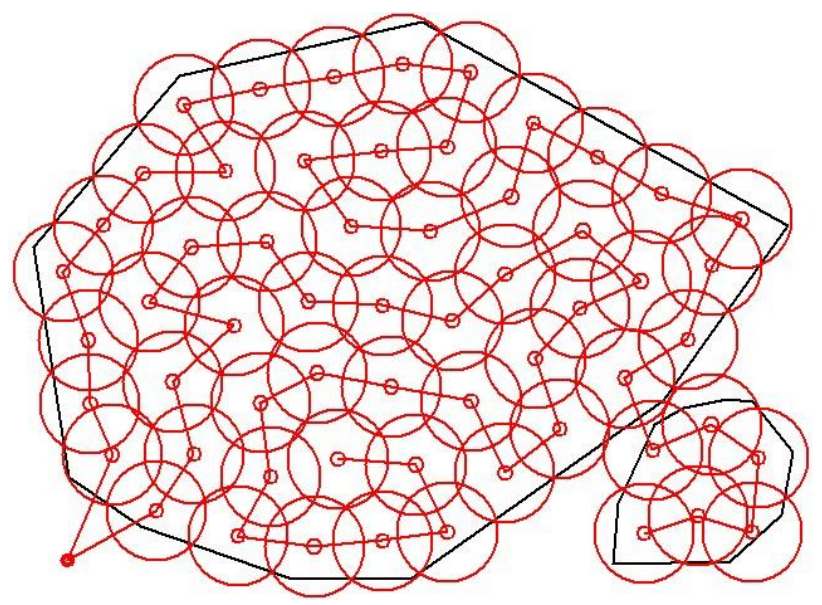

Fig. 2. Networks for 57 circles (case 2)

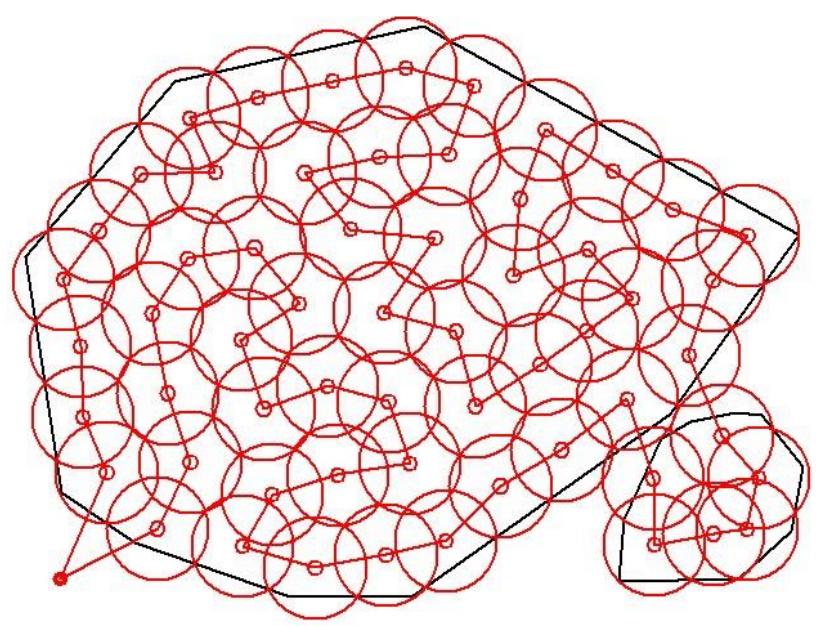

Fig. 3. Networks for 57 circles (case 3) 


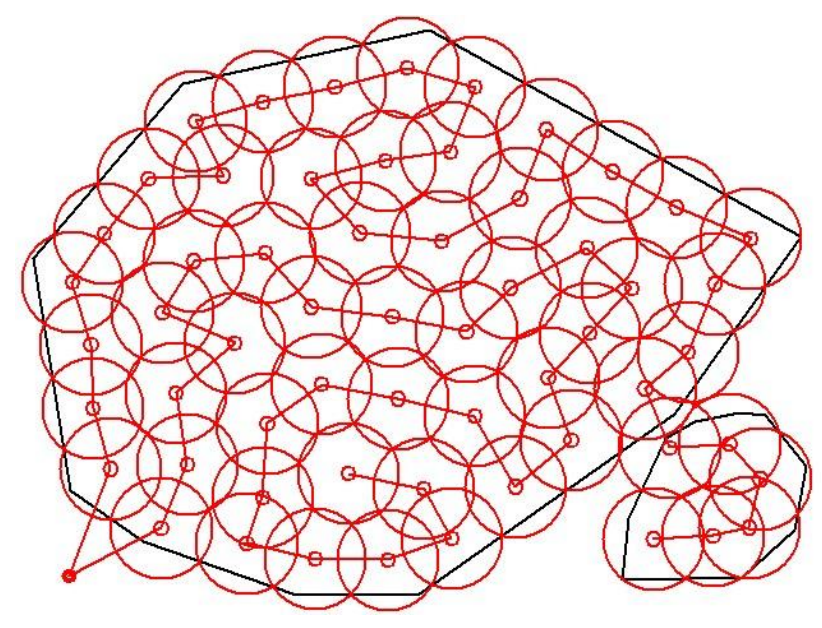

Fig. 4. Networks for 57 circles (case 4)

\section{REFERENCES}

[1] A. Sangwan and R. Singh "Survey on coverage problems in wireless sensor networks," in Wireless Personal Communications. 2015, no. 80(4), pp. 1475-1500.

[2] B. Wang "Coverage problems in sensor networks: A survey," ACM Comput. Surv. 2011, vol. 43, no. 4, p. 32.

[3] S. Shekhovtsov and S. Yakovlev "Formalization and solution of one class of covering problems in design of control and monitoring systems," in Avtomatika i Telemekhanica. 1989, no. 50(5), pp. 705710 .

[4] S. Yakovlev, O. Kartashov, V. Komyak, S. Shekhovtsov, O. Sobol and I. Yakovleva "Modeling and simulation of coverage problem in geometric design systems," Proc. of 15th International Conference on the Experience of Designing and Application of CAD Systems, CADSM 2019, pp. 20-23.

[5] A. So and Y. Ye "On solving coverage problems in a wireless sensor network using Voronoi diagrams," Int. Netw. Econ. 2005, no. 3828, pp. 584-593.

[6] H. Chizari, M. Hossein, T. Poston et al. "Delaunay triangulation as a new coverage measurement method in wireless sensor network," in Sensors (Basel). 2011, vol. 11(3), pp. 3163-3176.

[7] L. Lazos and R. Poovendran "Stochastic coverage in heterogeneous sensor networks," in ACM Trans. Sensor Netw. 2006, no. 2(3), pp. 325-358.

[8] P. Hall "Introduction to the Theory of Coverage Processesl," John Wiley \& Sons Incorporated. 1988, p. 432.

[9] X. Liu and D. He "Ant colony optimization with greedy migration mechanism for node deployment in wireless sensor networks," in J. Netw. Comput. Appl. 2014, vol.39, pp.310-318.

[10] L. Xunbo and W. Zhenlin "Cellular genetic algorithms for optimizing the area covering of wireless sensor networks," in C. J. of Electronics. 2011, vol. 20(2), pp. 352-356.
[11] M. Lanza, A. Gutierrez and J. Perez "Coverage optimization and power reduction in SFN using simulated annealing," in IET Communications. 2014, vol. 60(3), pp. 474-485.

[12] T. Tarnai and Zs. Gaspar "Covering a square by equal circles," in Elem. Math. 1995, vol. 50, pp. 167-170.

[13] V. Brusov and S. Piyavskij "Computational algorithm for the optimal coverage of plane areas," Computational Mathematics and Mathematical Physics. 1971, vol. 11(2), pp. 304-312.

[14] H. Jandl and K. Wieder "A continuous set covering problem as a quasidifferentiable optimization problem," in Optimization. 1988, vol. 19(6), pp. 781-802.

[15] E. Kiseleva, L. Lozovskaya and E. Timoshenko "The solution of continuous problems of optimal covering by spheres using the theory of optimal partitioning of sets," in Cybernetics and systems analysis. 2009, no. 3, pp. 98-117.

[16] P. Stetsyuk "Shor's r-algorithms: theory and practice". In: Optimization Methods and Applications: In Honor of the 80th Birthday of Ivan V. Sergienko. Butenko S., Pardalos P.M, Shylo V (Eds.). New York: Springer. 2017, pp. 495-520.

[17] V. Ushakov and P. Lebedev "Algorithms for optimal coverage of sets in the plane $\mathrm{R}^{2}$ ", in The Bulletin of Udmurt University. Mathematics. Mechanics. Computer Science. 2016, vol. 26(2), pp. 258-270.

[18] Y. Stoyan and V. Patsuk "Covering a compact polygonal set by identical circles," in Computational Optimization and Applications. 2010, vol. 46(1), pp. 75-92.

[19] V. Komyak, A. Pankratov, V. Patsuk and A. Prikhodko "The problem of covering the fields by the circles in the task of optimization of observation points for ground video monitoring systems of forest fires," in An international quarterly journal. 2016, vol. 5(2), pp. 133138.

[20] O. Antoshkin and A. Pankratov "Construction of optimal wire sensor network for the area of complex shape," in Eastern-European Journal of Enterprise Technologies. 2016, vol. 6(4), pp. 45-53.

[21] Yu. Stoyan, A. Pankratov and T. Romanova "Cutting and Packing problems for irregular objects with continuous rotations: mathematical modeling and nonlinear optimization," J. Oper. Res. Soc., 2016, vol. 67(5), pp 786-800.

[22] Yu. Stoyan, A. Pankratov, T. Romanova and A. Chugay "Optimized object packings using quasi-phi-functions," in Fasano G., Pinter J.D, (Eds.), Optimized Packings and Their Applications, New York: Springer. 2015, pp. 265-291 (Ser. Springer Optimization and Its Applications No. 105).

[23] G. Dantzig, R. Fulkerson and S. Johnson "Solution of a large-scale traveling salesman problem," in Operations Research. 1954, vol. 2(4), pp. 393-410.

[24] C. Groër, B. Golden and E. Wasil "A library of local search heuristics for the vehicle routing problem," in Mathematical Programming Computation. 2010 vol. 2(2), pp. 79-101.

[25] A. Wachter and L. Biegler "On the implementation of an interiorpoint filter line-search algorithm for large-scale nonlinear programming," in Mathematical Programming. 2006, no. 106(1), pp. $25-57$. 